\title{
Teaching cardiac auscultation to trainees in internal medicine and family practice: Does it work? B Favrat*, A Pécoud and A Jaussi
}

\author{
Address: Division of Cardiology and Outpatient Clinic, University Hospital of Lausanne Switzerland \\ Email: B Favrat* - Bernard.Favrat@hospvd.ch; A Pécoud - Alain.Pecoud@hospvd.ch; A Jaussi - andres.jaussi@vtx.ch \\ * Corresponding author
}

Published: 3I March 2004

BMC Medical Education 2004, 4:5

This article is available from: http://www.biomedcentral.com/l472-6920/4/5

(c) 2004 Favrat et al; licensee BioMed Central Ltd. This is an Open Access article: verbatim copying and redistribution of this article are permitted in all media for any purpose, provided this notice is preserved along with the article's original URL.

\begin{abstract}
Background: The general proficiency in physical diagnostic skills seems to be declining in relation to the development of new technologies. The few studies that have examined this question have invariably used recordings of cardiac events obtained from patients. However, this type of evaluation may not correlate particularly well with bedside skills. Our objectives were I) To compare the cardiac auscultatory skills of physicians in training with those of experienced cardiologists by using real patients to test bedside diagnostic skills. 2) To evaluate the impact of a five-month bedside cardiac auscultation training program.
\end{abstract}

Methods: I) In an academic primary care center, 20 physicians (trainees in internal medicine and family practice) and two skilled academic cardiologists listened to 33 cardiac events in 13 patients directly at bedside and identified the cardiac events by completing an open questionnaire. Heart sounds, murmurs and diagnosis were determined beforehand by an independent skilled cardiologist and were validated by echocardiography. Thirteen primary cardiologic diagnoses were possible.

2) Ten of the physicians agreed to participate in a course of 45-minute sessions once a week for 5 months. After the course they listened again to the same patients (pre/post-interventional study).

Results: I) The experts were the most skillful, achieving $69 \%$ recognition of heart sounds and murmurs and correct diagnoses in $62 \%$ of cases. They also heard all of the diastolic murmurs. The residents heard only $40 \%$ of the extra heart sounds and made a correct diagnosis in $24 \%$ of cases. 2) After the weekly training sessions, their mean percentage for correct diagnosis was $35 \%$ [an increase of $66 \%(p<0.05)]$.

Conclusions: The level of bedside diagnostic skills in this relatively small group of physicians in training is indeed low, but can be improved by a course focusing on realistic bedside teaching.

\section{Background}

Cardiac auscultation is considered as an important tool for doctors in obtaining a precise cardiovascular evaluation and ensuring a cost-effective approach in outpatientbased medicine [1-6].
Several recent studies evaluating the performance of clinicians in training have shown disappointing results for cardiac auscultation [7-10]. All of these studies, however, assessed the auscultatory performance of clinicians by using exclusively recordings of events obtained from patients. But since it does not test the real bedside diagnostic skills, this kind of evaluation may not be 
appropriate. Furthermore, even though medical educators are calling for additional teaching [11], we do not have any information about the possible impact of a bedside training program. The purpose of this study was to test, at the bedside, the clinical auscultation skills of trainees in internal medicine and family practice and to evaluate the impact of a 5-month bedside training program (45 min per week).

\section{Methods}

\section{First part}

Twenty physicians involved in an internal medicine and family practice training program at Lausanne University Outpatient Clinic in Switzerland participated in the study on a voluntary basis. We tested their auscultatory skills by using real patients, while two skilled academic cardiologists with considerable teaching experience in cardiology agreed to participate in the study as experts. Both participants and experts were blinded to the results of the physical examination results and echocardiography.

Each resident listened to 33 cardiac events that were considered to be key signs in 13 patients (10 men, 3 women, aged between 24 and 78 yrs) directly at the bedside (table 1 ). The patients lived in a remote area and were unknown to the participants. Heart sounds, murmurs and diagnoses were determined beforehand by an independent experienced cardiologist and validated by echocardiography. The patients were selected from those attending his private practice. The main echocardiographically confirmed diagnoses to be identified were aortic regurgitation [3], aortic stenosis [1], mitral regurgitation [4], mitral stenosis [2], LV dysfunction [3], pulmonary hypertension with tricuspid regurgitation [1], surgically corrected tetralogy of Fallot [1], ductus arteriosus [1], and ventricular septal defect [1]. Thus, the key signs to identify were abnormalities of normal heart sounds S1/S2, systolic and diastolic murmurs as well as ejection click, opening snap and additional diastolic sounds S3/S4.

During an afternoon session, the residents were asked to examine each patient for 10 minutes. The only information provided in each case was the patient's age and blood pressure. They were allowed to change the position of the patient as desired to improve auscultation. They then completed an open questionnaire asking them to describe their auscultatory findings and to state the precise diagnosis. Prior to examining the patients, the participants also completed a questionnaire designed to assess the importance they attributed to cardiac auscultation and the subjective self assessment of their auscultatory skills. None of the participants had a hearing problem.

\section{Second part}

Because of conflicting schedules, ten of the 20 participants were able to participate in a 45-minute course once a week for 5 months (Table 1). An experienced cardiologist taught the course using other current patients, one or two each week, with similar main cardiological diagnoses.

After the course, the participants listened again to the same patients according to the pre/post-interventional study design. None of the patients experienced any major changes during this period, either as regards their cardiac pathology or their auscultatory findings as assessed by the independent cardiologist. The participants were blinded to the final diagnoses until completion of the last part of the study.

\section{Statistical analysis}

The major questions assessed were: 1) What is the difference between experts and residents in respect of auscultation and diagnostic skills? 2. Is any improvement possible with a bedside auscultation training program?

One point was scored for each diagnosis or key sign correctly identified. The maximum scores were 33 and 13 for key signs and diagnoses respectively. The results for key signs and diagnoses were reported as accuracy rates and expressed as the percentage of total number of events or diagnoses identified by each participant (mean scores \pm $\mathrm{SD}$ in parentheses). Student's t-test was used to compare the experts with the residents. The univariate paired Student's t-test was used to compare the results of the residents before and after training. A p value of less than 0.05 was considered significant. Correlations between self-confidence in auscultation and auscultatory accuracy were analyzed by Spearman rank correlation.

\section{Results}

The two experts recognized $69 \%$ of the key signs vs. $40 \%$ \pm 10 for the whole group of twenty residents and correctly identified $62 \%$ of the diagnoses confirmed by echocardiography vs. $24 \% \pm 14$ (range $8 \%$ to $46 \%$ ) for the twenty residents. The experts heard $100 \%$ of the diastolic murmurs vs. $62 \%$ for the residents. Figure 1 and 2 show the percentage of recognized key signs and the percentage of correct diagnosis for all participants.

The 10 residents involved in the regular teaching program participated in at least 10 sessions (mean $14.6 \pm 5.1$ ). They recognized $42 \% \pm 10$ of the heart sounds and murmurs both before and after training, but gave the correct diagnosis in only $21 \% \pm 15$ of cases before training, compared to $35 \% \pm 17$ after training, a relative increase of $66 \%(\mathrm{p}=$ 0.03 ) (fig. 3). They recognized $64 \%$ of diastolic murmurs vs. $78.5 \%$ after the training ( $\mathrm{p}=0.047)$, they heard ejection clicks in $22 \%$ before vs. $45 \%$ after the training ( $\mathrm{p}=$ 
Table I: Characteristics of participating physicians

\begin{tabular}{lll}
\hline & All & Study group \\
\hline N & 20 & 10 \\
Age (yrs) & $33.5 \pm 7$ & $32.3 \pm 4.5$ \\
Postgraduate training & $4.4 \pm 2.5$ & $3.9 \pm 2.5$ \\
(internal medicine & $2.9 \pm 1.6$ & $2.7 \pm 2)$ \\
Importance attributed to physical examination(I-4) & $3.3 \pm 0.6$ & $3.4 \pm 0.7$ \\
Self-assessment (I-3) & $2.0 \pm 1$ & $2.2 \pm 1$ \\
Participation in specific training (hours) & & $14.6 \pm 5.1$ \\
+ 2 EXPERTS (VERY EXPERIENCED CARDIOLOGISTS) & & \\
\hline
\end{tabular}

"study group" = physicians having participated in the five-month training program Plus-minus values are means \pm SD. No statistical significance was found.

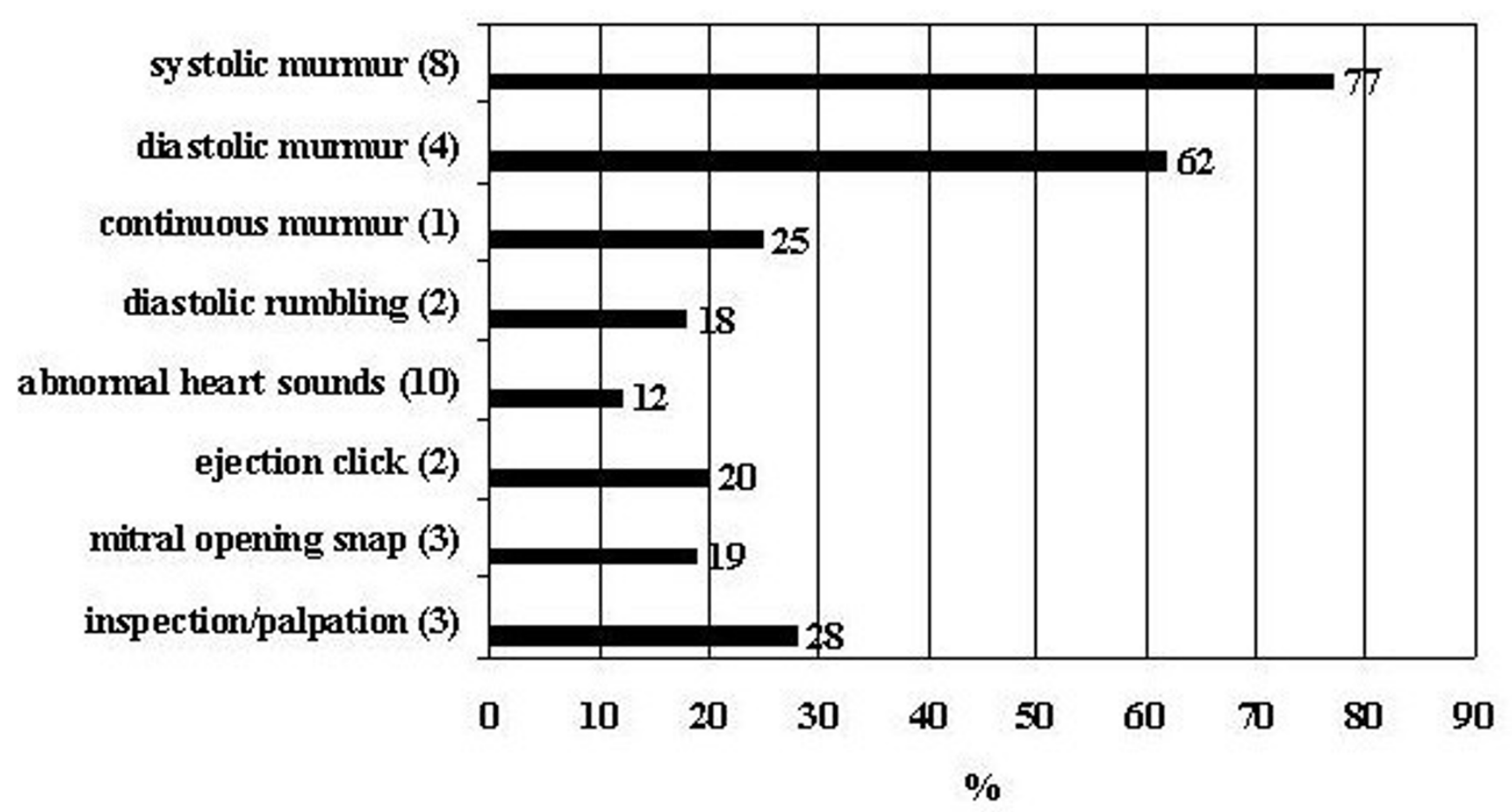

Figure I

Percentage of recognized "key signs" for all participants

$0,02)$. The main areas of improvement for diagnostic accuracy rates were aortic insufficiency $(11 \% \pm 22$ before vs. $40 \% \pm 26)$ after training, $\mathrm{p}=005)$ and aortic stenosis (55\% vs. $80 \%$, p < 0.05). The experts recognized all aortic regurgitations and stenoses.

We found no significant correlation between self-confidence in auscultation and auscultatory accuracy $r=0.21$ (Figure 4). Subgroup analysis was not possible due to the small number of participants.

\section{Discussion}

We found a substantial difference in auscultatory performance between experts and residents under the same conditions at the bedside. All residents had great difficulty in recognizing important diagnoses such as aortic regurgitation. The level of bedside diagnostic skills was very low. The original feature of this study was the use of real patients. In fact, we consider that this methodology offers the ideal opportunity for creating a more realistic situation than cardiac tapes or mannequins. Nonetheless, we 


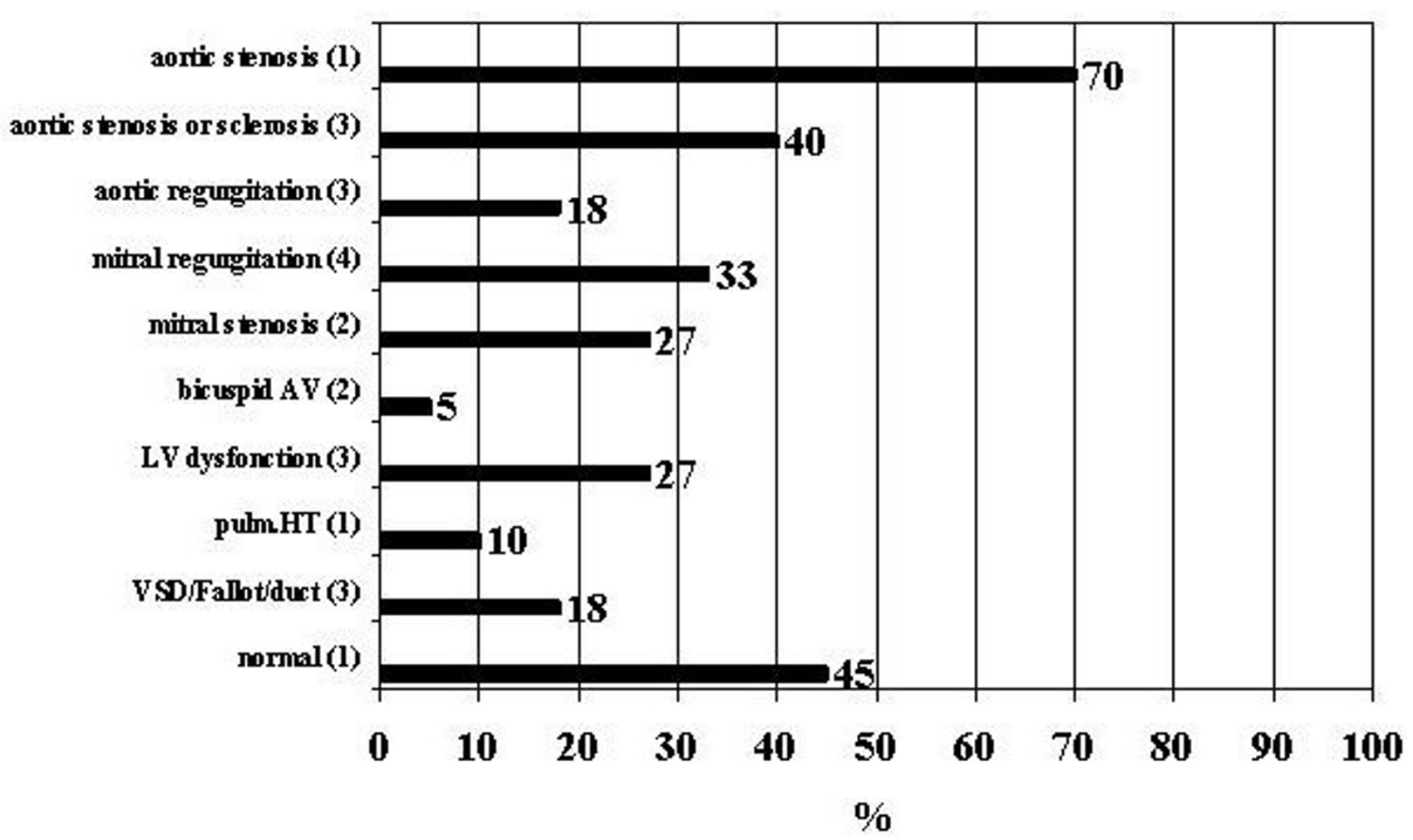

Figure 2

Percentage of correct diagnosis for all participants

found the same low level of diagnostic skill observed in previous studies for trainees in internal medicine or family practice: residents in pediatrics found a diagnostic accuracy of 33\% using an adult size mannequin [8] and trainees in internal medicine and family practice recognized $20 \%$ of auscultatory events in a study using tape recordings [7]. Furthermore, our study provides information about the considerable gap between experts and residents. Obviously, one of the major objectives of any training program should be the narrowing of this gap.

Auscultation skills are better evaluated in real conditions where the elimination of contaminating background noise is not always possible, in contrast with a perfect audiotape recording. Furthermore, the bedside testing includes the option of changing the position of the patient, thus allowing the doctor to assess the radiation pattern of murmurs and the change in character of the murmur. The gold standard was the echocardiography and therefore, as expected, even the experts were unable correctly to identify all the diagnoses. Residents managed to identify about half of what an expert can achieve. Interestingly, the performance levels observed for expert cardiologists with an audiotape range from 80 to 90 percent
[7], compared to $62 \%$ in our study. This study confirms that a low level of proficiency in cardiac auscultation is found not just in English-speaking countries [9] but also in others, including French-speaking countries. So this finding is not related to language, but rather, as suggested by Mangione [9] to the rapid and unlimited access to sophisticated technology in the western world, which gives the false illusion that a precise clinical diagnosis is unnecessary or useless but just time-consuming.

We found that an improvement in diagnostic skills could be achieved by means of a training program consisting of bedside auscultation 45 minutes once a week over a 5month period. No significant improvement was noted in this study in the identification of sounds and murmurs by auscultation alone, which suggests that a bedside teaching course could lead mainly to a better interpretation of the sounds and murmurs being heard. Nevertheless, this training offers encouraging prospects because it shows that more frequent exposure to patients in a didactic setting can improve diagnostic skills of physicians during postgraduate training. 


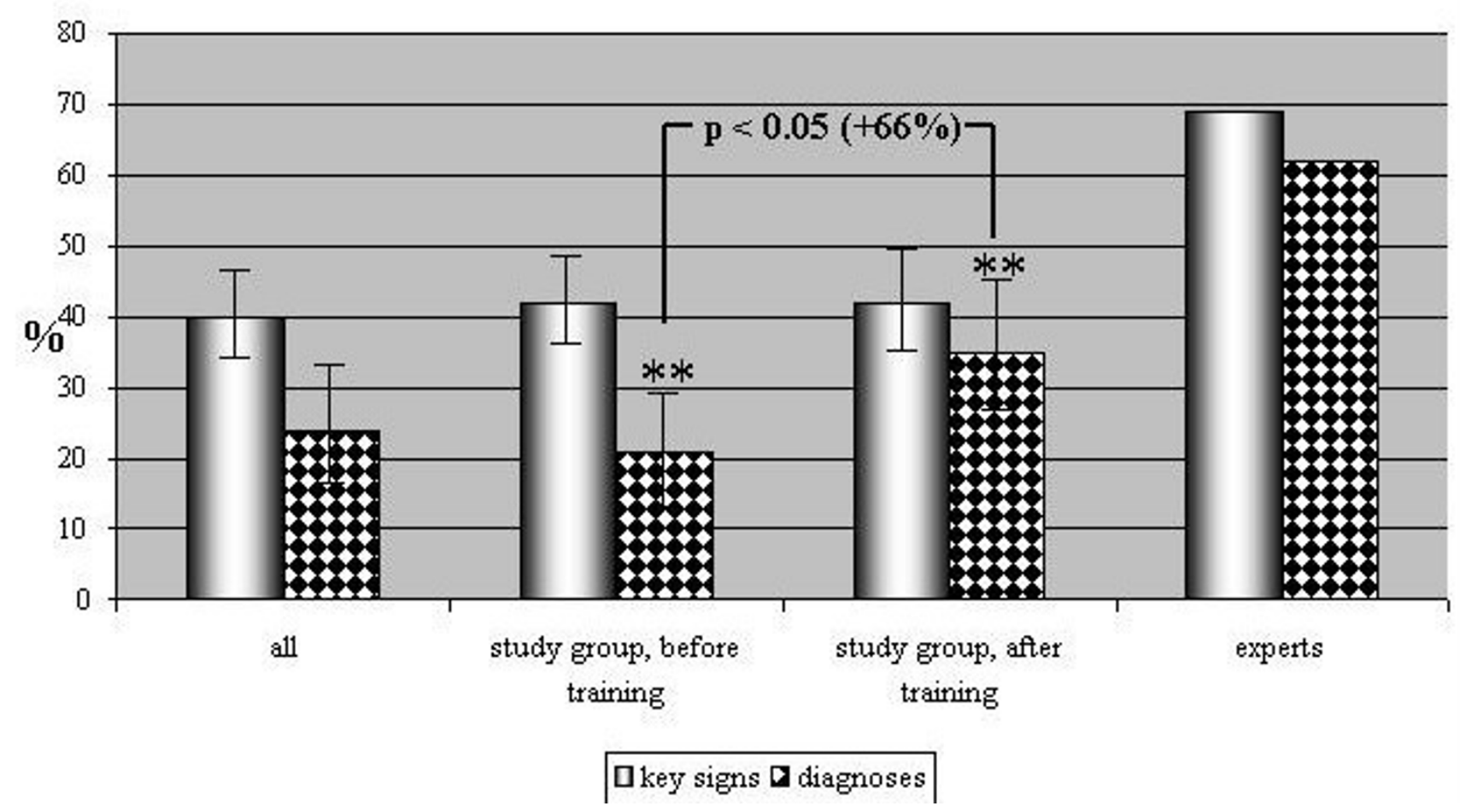

Figure 3

Accuracy of physical examination

In an era of increasing awareness of cost effectiveness, physicians are having to decide how to avoid both the overuse and underuse of instrument-based examinations. Obviously, this decision will essentially depend on the quality of the clinical evaluation. In our case, residents were clearly less skillful than experts in making important diagnoses, e.g. diastolic murmurs, which were not heard by the trainees in $60 \%$ of cases. One could argue that the interpretation of auscultatory findings could prove difficult without a detailed clinical history. But in the case of bicuspid aortic valve, for example, a "minor" congenital malformation with the potential for serious long-term consequences, there is usually no significant history, and the screening and clinical diagnosis - and thus the indication for further important investigation - depend solely on the auscultation skill of physicians. Such missed cases could prove detrimental to the patients concerned. By contrast, overdiagnosis could lead to greater and inappropriate utilization of resources and higher care costs. Referral should always be appropriate. Obviously, a further erosion of skills will lead to greater use of technol- ogy to compensate for the growing lack of clinical knowledge, but also to increasing numbers of missed patients who actually need to be referred. Only a structured course and a real emphasis on auscultatory skills during postgraduate training will help to improve this situation.

Finally, it is notable that self-confidence was not a predictor of success. This shows how residents have an inaccurate perception of their own abilities in this field, and also highlights the need for an improvement in teaching by giving feed-back to trainees to correct their self perception. Hopefully, this could avoid over-generous self-assessments and a consequent underestimation or overestimation of the risks inherent in a clinical situation.

An important limitation of this study was the absence of a clear-cut control group. We considered the participants to be their own controls before and after the training. Among the 20 trainees, 10 were not able to participate in the intervention and could not be used as a control group because of the high turn over of our training site. Though 


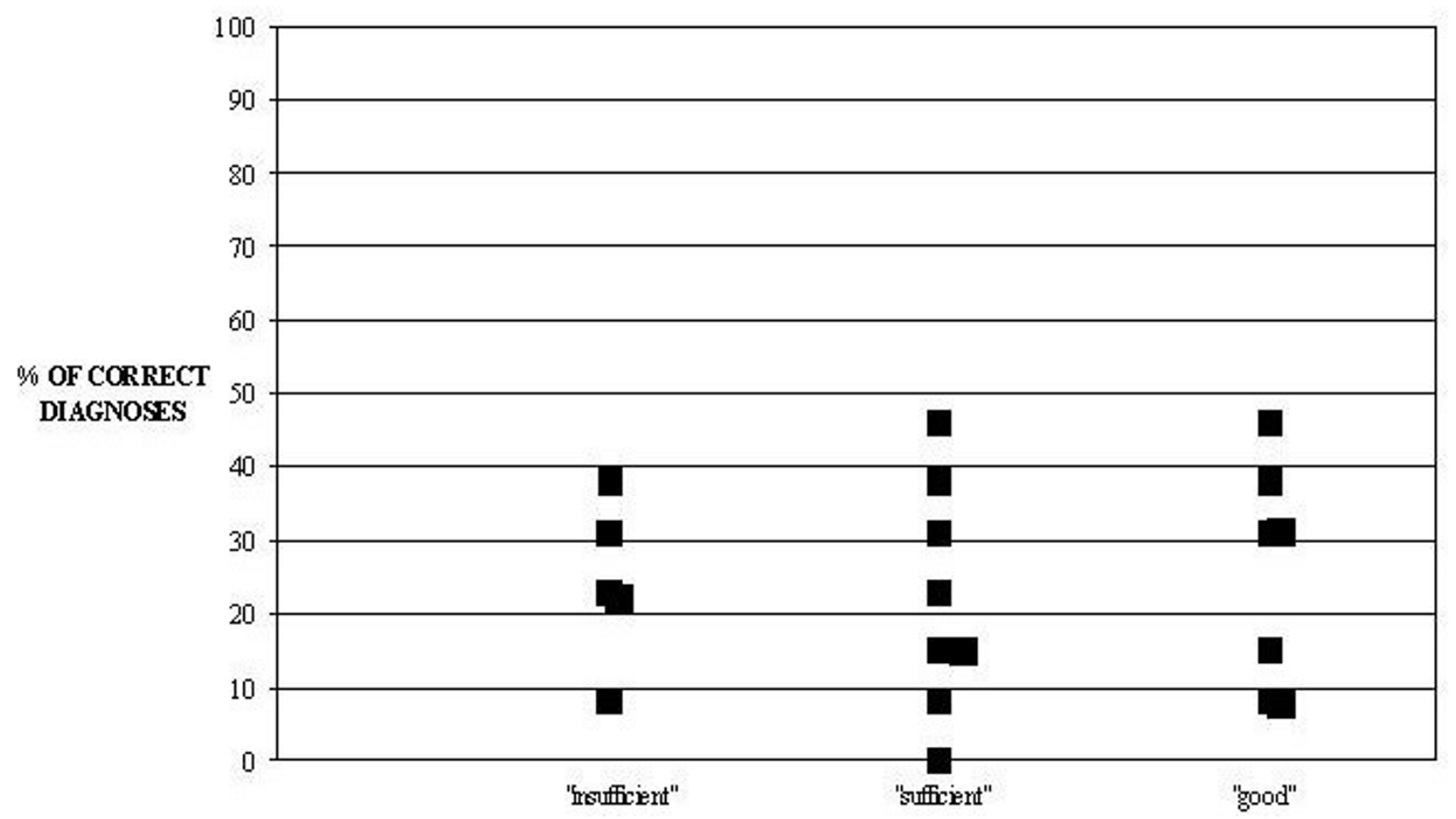

Figure 4

Self-assessment of the participants

when the control group is not randomly selected, validity of the data is also questionable. We decided not to conduct a formal randomized clinical trial, believing it to be unethical to refuse the teaching course to residents during such a relatively long period of their postgraduate training. However, because no study was found using real patients, such a prospective observational study can be useful [12]. A recent study [13] with 15 residents using simulated heart sounds found an improvement with a teaching course in the same pre/post intervention design but the author considered as an important limitation the use of sounds from a stimulator and not from real patients. Our study gives data for that last point and is original in that perspective. A second limitation is the small sample size of physicians involved in this study and therefore it is difficult to draw broad generalisations from the study. It would be of interest to have similar studies repeated at other institutions with real patients as well.

\section{Authors' contributions}

$\mathrm{BF}, \mathrm{AJ}, \mathrm{AP}$ participated in the conception and design of the study. Analysis, interpretation of data, drafting, revising the manuscript and inclusion of patients for BF and AJ.
Table 2: Characteristics of patients

$\begin{array}{lr}33 \text { clinical signs considered as important or « key signs » main } & \\ \text { diagnoses: } & 3 \\ \text { aortic regurgitation } & \text { I } \\ \text { aortic stenosis } & 4 \\ \text { mitral regurgitation } & 2 \\ \text { mitral stenosis } & 3 \\ \text { left ventricular dysfunction } & \text { I } \\ \text { pulmonary hypertension with tricuspid regurgitation } & \text { I } \\ \text { ventricular septal defect } & \text { I } \\ \text { operated Tetralogy of Fallot } & \text { ductus Botalli }\end{array}$

13 patients (10 men, 3 women; 24-78 yrs.)

\section{Acknowledgements}

None

\section{References}

I. Kern DC, Parrino TA, Korst DR: The lasting value of clinical skills. JAMA 1985, 254:70-76.

2. Peterson MC, Holbrook JH, Hales DV, Smith NL, Staker LV: Contributions of the history, physical examination, and laboratory investigation in making medical diagnoses. West J Med 1992, 156:163-165. 
3. Fletcher RH, Fletcher SW: Has medicine outgrown physical diagnosis? Ann Intern Med 1992, I I 7:786-787.

4. Craige E: Should auscultation be rehabilitated? $N$ Engl J Med 1988, 318:1611-1613.

5. Weitz HH, Mangione $S$ : In defense of the stethoscope and the bedside. Am J Med 2000, 108:669-67I.

6. Adolph RJ: In defense of the stethoscope. Chest 1998 I 14:1235-1237.

7. St Clair EW, Oddone EZ, Waugh RA, Corey GR, Feussner JR: Assessing housestaff diagnostic skills using a cardiology patient simulator. Ann Intern Med 1992, I I7:751-756.

8. Mangione S, Nieman LZ: Cardiac auscultation skills of internal medicine and family practice trainees: a comparison of diagnostic proficiency. JAMA 1997, 278:717-722.

9. Gaskin PR, Owens SE, Talner NS, Sanders SP, Li JS: Clinical auscultation skills in pediatric residents. Pediatrics 2000, 105: I184-1187.

10. Mangione S: Cardiac auscultatory skills of physician-in-training: A comparison of three English speaking countries. $\mathrm{Am} J$ Med 200I, I 1 0:210-6.

II. Marcus FI: The lost art of auscultation. Arch Intern Med 1999, 159:2396.

12. Black N: Why we need observational studies to evaluate the effectiveness of health care. BMJ 1996, 312:1215-1218.

13. Horiszny JA: Teaching Cardiac Auscultation Using Simulated Heart Sounds and Small-group Discussion. Fam Med 200I, 33:39-44.

\section{Pre-publication history}

The pre-publication history for this paper can be accessed here:

http://www.biomedcentral.com/1472-6920/4/5/prepub

Publish with Biomed Central and every scientist can read your work free of charge

"BioMed Central will be the most significant development for disseminating the results of biomedical research in our lifetime. "

Sir Paul Nurse, Cancer Research UK

Your research papers will be:

- available free of charge to the entire biomedical community

- peer reviewed and published immediately upon acceptance

- cited in PubMed and archived on PubMed Central

- yours - you keep the copyright 